\title{
TENDÊNCIAS DE EXTREMOS CLIMÁTICOS NA REGIÃO DE TRANSIÇÃO AMAZÔNIA-CERRADO NO ESTADO DO MARANHÃO
}

\author{
SANTOS, Jessflan Rafael Nascimento - jessflan@gmail.com \\ Universidade CEUMA / UniCEUMA
}

\author{
ARAÚJO, Mayara Lucyanne Santos de - mayara.araujo.eng@gmail.com \\ Universidade Federal de Campina Grande / UFCG \\ SILVA JUNIOR, Celso Henrique Leite - celso.junior@inpe.br \\ Instituto Nacional de Pesquisas Espaciais / INPE
}

\author{
SANTOS, Juliana Sales dos - julianasales.engenharia@gmail.com \\ Universidade CEUMA / UniCEUMA \\ ALMEIDA, Juliana Lopes - july01.jl@gmail.com \\ Universidade CEUMA / UniCEUMA \\ LIMA, Taynara Viana - taynaralima48@gmail.com \\ Universidade CEUMA / UniCEUMA \\ SOUSA, Luan Victor Pereira de - luanv1996@gmail.com \\ Universidade CEUMA / UniCEUMA \\ AGUIAR, Pedro Henrique Modesto de - phm.aguiar18@gmail.com
Universidade CEUMA / UniCEUMA \\ SILVA, Fabrício Brito - fabricioagro@gmail.com \\ Universidade CEUMA / UniCEUMA
}

\begin{abstract}
RESUMO: Os extremos climáticos têm sido um assunto de grande interesse na pesquisa sobre mudanças climáticas, pois seu entendimento fornece subsídios para 0 gerenciamento de eventos que podem levar a desastres naturais e desequilíbrios nos sistemas sociais e produtivos. Assim, este trabalho teve como objetivo estimar extremos derivados da temperatura e precipitação (coletados por estações meteorológicas) na região de transição Amazônia-Cerrado do estado do Maranhão no período de 1986 a 2016. O software RClimdex e o teste estatístico não-paramétrico de Mann-Kendall foram utilizados para detectar tendências de aumento ou diminuição nas variáveis analisadas. Verificou-se que as temperaturas máxima e mínima tiveram uma tendência significativa de aumento $(p<0,05)$, revelando que os dias e as noites estão sendo mais quentes em todo o estado no período analisado. Em relação à chuva, foi encontrada alta variabilidade espaço-temporal, sem um padrão de tendência claro. No entanto, a estação meteorológica de Carolina (Cerrado) teve uma tendência de aumento de chuva significativa $(p<0,05)$, enquanto a estação meteorológica de Zé Doca (Amazônia) teve uma tendência de redução significativa de chuva $(p<0,05)$. Assim, conclui-se que o clima está mudando de maneira diferente no estado do Maranhão, sendo necessária a elaboração de políticas para adaptar a região a essas mudanças.
\end{abstract}

PALAVRAS-CHAVE: mudanças globais, variabilidade climática, vulnerabilidade climática, nordeste brasileiro.

TRENDS OF CLIMATE EXTREMES IN THE AMAZON-SAVANNA TRANSITION REGION IN THE MARANHÃO STATE 
ABSTRACT: Climate extremes have been a subject of great interest in climate change research, as their understanding provides insights for managing events that may lead to natural disasters and imbalances in social and productive systems. Thus, this work aimed to estimate extremes derived from temperature and rainfall (collected by weather stations) in the Amazon-Cerrado transition region of the Maranhão state within the 19862016 period. The software RClimdex and the nonparametric Mann-Kendall statistical test were used to detect the increase or decrease trends in the analyzed variables. It has been found that the maximum and minimum temperatures show a significant upward trend $(p<0.05)$, revealing that the days and nights are being warmer throughout the state in the analyzed period. Regarding rainfall, high spatiotemporal variability was found, without a clear trend pattern. However, the Carolina weather station (Cerrado) had a significant rainfall increase trend $(p<0.05)$, while the Zé Doca weather station (Amazon) had a significant rainfall decrease trend $(p<0.05)$. Thus, it is concluded that the climate is changing differently thorough the Maranhão state, needed the elaboration of policies to adapt the region to these changes.

KEYWORDS: global changes, climate variability, climate vulnerability, brazilian northeast.

\section{INTRODUÇÃO}

Diversos estudos têm evidenciado as mudanças climáticas por meio de avaliações do comportamento médio de variáveis meteorológicas em diferentes escalas temporais e espaciais (GUO et al., 2017; IPCC, 2013; GOCIC e TRAJKOVIC, 2013). Porém, pequenas variações nos valores médios nos elementos do clima podem estar associadas a grandes mudanças na frequência de eventos extremos (KATZ e BROWN, 1992). Por isso, em estudos recentes, as investigações sobre mudanças no clima têm avaliado, além do comportamento médio, os extremos climáticos (SHI et al., 2018; YE et al., 2018).

Em escalas regional e local, mudanças no clima associadas ao aumento de eventos extremos têm sido evidenciadas em diferentes magnitudes (IPCC, 2012, LÜ et al., 2018; GUMMADI et al., 2017; HASAN e SALLEH, 2017; MUTIIBWA et al., 2015). Esses eventos têm sido tema de grande interesse em pesquisas de mudanças climáticas por proporcionar subsídios para gestão de eventos de desastres naturais e planejamento frente aos desequilíbrios nos sistemas sociais e produtivos (ROBINNE et al., 2018; KUNWAR e BOHARA, 2017; YANG e XU, 2017).

Segundo a Organização Mundial de Meteorologia (OMM), mais de 370 mil pessoas ao redor do mundo morreram decorrente das condições do clima e dos extremos climáticos, devido ao aumento da ocorrência de ondas de calor, períodos de frio, secas, tempestades e inundações no período de 2001-2010 (WMO, 2013). No Brasil, estudos têm evidenciado mudanças climáticas através de analises de tendências para detecção de extremos climáticos em diferentes escalas (ASSIS, LACERDA e SOBRAL, 2012; PEDRON et al., 2017), porém, as regiões de transição entre biomas e ecossistemas têm sido pouco exploradas.

A região de transição entre os biomas Amazônia e Cerrado possui particularidades ambientais diretamente impactadas pelas mudanças climáticas. Um exemplo desses impactos é a influência dos fenômenos de interação oceano-atmosfera na região de transição Amazônia-Cerrado, no Maranhão (FERREIRA \& MELO, 2005; ASSIS et al., 2015). O estado do Maranhão está situado na zona de transição Amazônia-Cerrado (IBGE, 2019) e é caracterizado pela alta diversidade de ecossistemas e consequente biodiversidade. Por 
exemplo, o Maranhão possui três sítios RAMSAR: Área de Proteção Ambiental das Reentrâncias Maranhenses, Área de Proteção Ambiental Baixada Maranhense e Parque Estadual Marinho do Parcel de Manuel Luis e Baixios do Mestre Álvaro e Tarol, estabelecidos pela convenção das Nações Unidas sobre a conservação da biodiversidade em áreas de trópicos úmidos (RAMSAR, 2018). Os mesmos constituem ecossistemas caracterizados por ciclos de inundações, com relevantes atividades econômicas e manifestações culturais relacionadas ao ambiente, com exceção do Parque Estadual Marinho do Parcel de Manuel Luis por ser uma Unidade de Conservação de Proteção Integral. Porém, apesar da vulnerabilidade dos ecossistemas em áreas de transição entre biomas, nenhum estudo abordou a tendência de ocorrência de eventos extremos climáticos na região do Maranhão, nem, tampouco, o consequente impacto nas propriedades ecossistêmicas, considerando também a dimensão humana.

Um aspecto pouco considerado em estudos climáticos é o detalhamento de como o clima está mudando, em escala temporal, indicando a magnitude da mudança em cada elemento do clima. Santos \& Oliveira (2017) relatam que no Estado do Pará, os dias e as noites estão mais quentes, onde o valor da temperatura mínima diária aumentou em taxas anuais, $0,046^{\circ} \mathrm{C}, 0,055^{\circ} \mathrm{C}$, $0,056^{\circ} \mathrm{C}$ e $0,048^{\circ} \mathrm{C}$ no verão, outono, inverno e primavera, respectivamente. Porém, não foram definidos limiares para configurar um extremo em relação à um valor médio de acordo com a região, assumindo o valor padrão definido pelo o programa RClimdex.

Nesse contexto, quatro questões climáticas permanecem não abordadas em estudos em regiões de transição Amazônia-Cerrado: 1) Estão ocorrendo eventos extremos que configurem a mudança do clima na região de transição Amazônia-Cerrado, no Maranhão? 2) Com que magnitude a temperatura e precipitação estão configurando as tendências de mudança climática? 3) Existe um padrão espacial definido para as mudanças climáticas nesta região estudada? 4) A ocorrência de eventos climáticos extremos é influenciada por anomalias da temperatura da superfície do mar nos oceanos Pacífico e Atlântico?

Assim, o objetivo deste trabalho é avaliar a tendência dos eventos de extremos climáticos referentes a temperatura e precipitação na região de transição Amazônia-Cerrado no estado do Maranhão.

\section{MATERIAIS E MÉTODOS}

\section{ÁREA DE ESTUDO}

O Estado do Maranhão abrange uma área de $331.935,5 \mathrm{~km} 2$ e sua população atual é de 6.574.789 habitantes (IBGE, 2010), correspondendo a uma densidade populacional de 19,81 hab.km-2, com $63,1 \%$ da população vivendo em áreas consideradas urbanas (4.147.149 habitantes).

O Maranhão se encontra em uma zona de transição entre os biomas Amazônico e Cerrado (figura 1). Dessa forma, se encontra numa posição privilegiada entre três macrorregiões brasileiras: Norte (Amazônia), Centro Oeste (Cerrado) e Nordeste (Semiárido), e, portanto, reúne características fitogeográficas e climatológicas características dessas áreas (Maranhão, 2002).

De acordo com o método de classificação climática de Köppen (ÁLVARES et al., 2013), o Maranhão possui três tipos climáticos: o Am - zona tropical com 
período monção (fevereiro a maio), caracterizado por apresentar a precipitação média do mês mais seco inferior a $60 \mathrm{~mm}$ e média total anual $\geq 3300 \mathrm{~mm}$, o As - regiões tropicais com o verão seco e o Aw - zona tropical com o inverno seco, abrangendo maior parte do estado, em que o período chuvoso mais acentuado acontece nos meses de dezembro, janeiro e fevereiro com a média mensal acima de $250 \mathrm{~mm}$. Todas as classificações possuem a temperatura acima ou igual a $18 \circ \mathrm{C}$ durante o ano todo.

\section{CONJUNTO DE DADOS}

Os dados climatológicos foram obtidos de 12 estações meteorológicas do Instituto Nacional de Meteorologia - INMET distribuídas no Estado do Maranhão. Foram utilizados registros diários de dados de precipitação, temperatura máxima e mínima para o período de 1986 a 2016 (Figura 1).

Os dados de anomalias da temperatura da superfície do mar (TSM) para os Oceanos Pacífico e Atlântico foram obtidos da Administração Nacional Oceânica e Atmosférica (NOAA), portal do Laboratório de Pesquisa do Sistema Terrestre. Foram utilizados 0 índice El Niño Multivariado - MEI (https://www.esrl.noaa.gov/psd/enso/mei/table.html), o índice de Oscilação Decadal do Pacífico - PDO (https://www.ncdc.noaa.gov/teleconnections/pdo/) e o índice de Oscilação Mutidecadal do Atlântico - AMO (https://www.esrl.noaa.gov/psd/data/timeseries/ AMO).

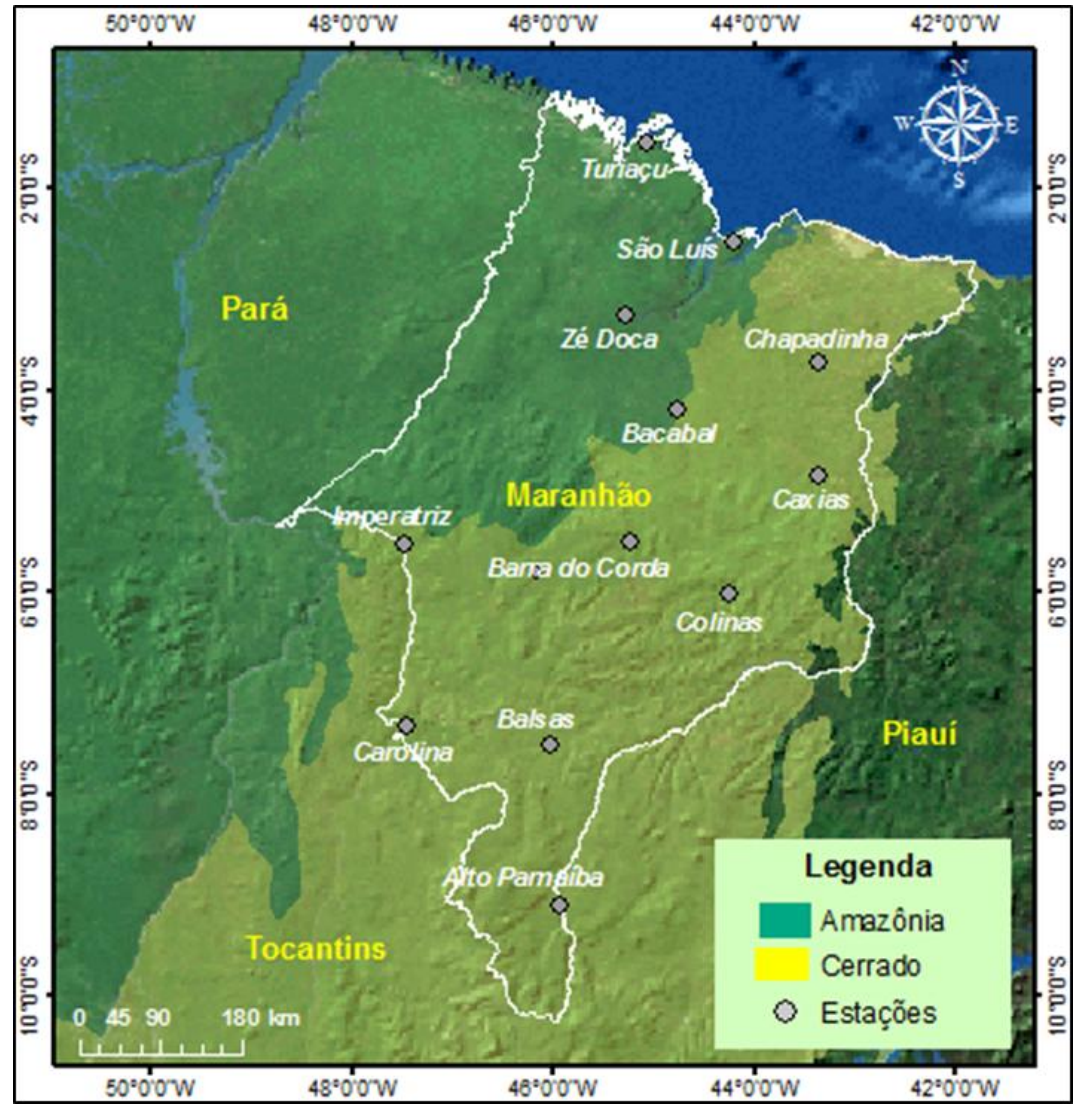

Figura 1 - Localização das 12 estações meteorológicas no Estado do Maranhão (INMET), destacando os biomas Amazônia e Cerrado segundo o IBGE. Fonte: Silva et al, 2016. 


\section{ESTIMATIVA DOS EXTREMOS CLIMÁTICOS}

Para obtenção dos índices de detecção e monitoramento de extremos de mudanças climáticas existentes no Estado do Maranhão foi utilizado o programa computacional RCLIMDEX (Versão 3.2.1).

O RCLIMDEX calcula 27 índices, sendo 11 índices de precipitação e 16 índices de temperatura propostos pela equipe de Especialistas em Detecção, Monitoramento e Índices de Mudanças Climáticas (ETCCDMI), foi desenvolvido por Xuebin Zhang e Feng Yang do Serviço Meteorológico Canadense (ZHANG e YANG, 2004). Neste trabalho foram utilizados apenas 11 índices para temperatura e 11 para precipitação (tabela 1 (a) e (b)).

Para os registros de dados serem reconhecidos pelo RCLIMDEX, os dados faltosos devem, necessariamente, ser codificados em -99.9 e os registros de dados devem estar em ordem cronológica.

A tendência anual foi obtida pelo método dos mínimos quadrados. 0 teste não paramétrico Mann-Kendall (Man, 1945; Kendall, 1975) foi utilizado para avaliar os sinais de tendência e a significância estatística dos índices de extremos climáticos. Seja uma série temporal de observações, x1, x2, x3, ..., $\mathrm{xn}$, representa $\mathrm{n}$ pontos em que $\mathrm{Xj}$ representa o ponto no tempo $\mathrm{j}$. A estatística (S) do teste de Mann-Kendall é calculada de acordo com a equação 1.

$$
S=\sum_{k=1}^{n_{i}-1} \sum_{j=k+1}^{n} \operatorname{sgn}\left(X_{j}-X_{k}\right)
$$

Em que:

$$
\operatorname{sgn}(x)=\left\{\begin{aligned}
+\mathbf{1}, & x>0 \\
\mathbf{0}, & x=0 \\
-\mathbf{1}, & x<0
\end{aligned}\right.
$$

Valores positivos de $\mathrm{S}$ indicam uma tendência de crescimento no qual os valores crescem com o tempo, e valores negativos indica uma tendência negativa. Contudo, é necessário computar a probabilidade associada com $\mathrm{S}$ e a dimensão da amostra, n, para estatisticamente quantificar a significância da tendência. Para uma dimensão da amostra $>10$, as aproximações normais para o teste de Mann-Kendall podem ser usadas.

A estatística $S$ está próxima da normal à medida que $n$ aumenta. A média, E, e a variância, V, são definidas nas equações 3 e 4:

$$
\begin{gathered}
E(S)=0 \\
V(S)=\frac{n(n-1)(2 n-1)-\sum_{j=1}^{p} t_{j}\left(t_{j}-1\right)\left(2 t_{j}+5\right)}{18}
\end{gathered}
$$


Em que $p$ é número de grupos contendo valores iguais na série de dados e $t j$ é o número de dados com valores iguais num certo grupo $j$.

Sabendo-se que $S$ é normalmente distribuído e tem média zero e variância dada pela Equação 1, pode se checar se a tendência positiva ou negativa é significante diferente de zero. Se $S$ é significante diferente de zero, Ho pode ser rejeitada para certo nível de significância apontando existência de tendência ( $H_{1}$ é aceita).

Mann (1945) e Kendall (1975) mostraram que mesmo para valores pequenos de $n$, pode-se assumir uma distribuição normal desde que o valor de estatística $Z$ seja dado por:

$$
Z(t)=\left\{\begin{array}{ccc}
\frac{S-1}{[V(S)]^{1 / 2}} & \text { se } & S>0 \\
0 & \text { se } & S=0 \\
\frac{S+1}{[V(S)]^{1 / 2}} & \text { se } & S<0
\end{array}\right.
$$

A estatística do teste $Z$ (t) é usada para medir a significância da tendência. Dessa forma, a estatística do teste é usada para testar a hipótese nula $H_{0}$. Se $Z$ (t) for maior que $Z a / 2$, onde a representa o nível de significância escolhido, então a hipótese nula é rejeitada, indicando que a tendência é significativa.

Tabela 1 - Lista dos 11 índices de extremos climáticos de temperatura (a) e 11 índices de precipitação (b) do ETCCDMI

(a) Índices de temperatura

\section{Nome do índice}

Percentil

Dias quentes

Dias frios

Noites quentes

Noites frias

\section{Limiares}

Dias de verão

Noites tropicais

Dias de verão

Noites tropicais

\section{ID}

Definição

Unidade

$\%$ de dias em que a Tmáx

$>90$ percentil

$\%$ de dias em que a Tmáx

$<10$ percentil

$\%$ de dias em que a Tmin

$>90$ percentil

$\%$ de dias em que a Tmin

Tn10p

$<10$ percentil

$\%$

$\%$

$\%$

$\%$
Número de dias em que a

Tmáx $>25^{\circ} \mathrm{C}$

Dias

Dias

Tmin $>20^{\circ} \mathrm{C}$

Número de dias em que a

SU

Tmáx $>X^{\circ} \mathrm{C}$ definido pelo

Dias usuário

TR Número de dias em que a Dias 
Tmin $>X^{\circ} \mathrm{C}$ definido pelo usuário

\section{Absoluto}

Dia mais quente

Dia mais frio

Noite mais quente

Noite mais fria

Variação de temperatura diária

$\begin{array}{lll}\text { TXx } & \begin{array}{l}\text { Valor máximo mensal da } \\ \text { Tmáx diária }\end{array} & { }^{\circ} \mathrm{C} \\ \text { TXn } & \begin{array}{l}\text { Valor mínimo mensal da } \\ \text { Tmáx diária }\end{array} & { }^{\circ} \mathrm{C} \\ \text { TNx } & \begin{array}{l}\text { Valor máximo mensal da } \\ \text { Tmin diária }\end{array} & { }^{\circ} \mathrm{C} \\ \text { TNn } & \begin{array}{l}\text { Valor mínimo mensal da } \\ \text { Tmin diária }\end{array} & { }^{\circ} \mathrm{C} \\ \text { DTR } & \text { Tmáx diária - Tmin diária } & { }^{\circ} \mathrm{C}\end{array}$

${ }^{\circ} \mathrm{C}$

${ }^{\circ} \mathrm{C}$

${ }^{\circ} \mathrm{C}$

${ }^{\circ} \mathrm{C}$

${ }^{\circ} \mathrm{C}$

(b) Índices de Precipitação

\section{Nome do índice}

Percentil

Dias muito úmidos

Dias extremamente

úmidos

ID

Definição

Unidade

\section{Limiares}

Precipitação de um dia superior a $10 \mathrm{~mm}$

Precipitação de um dia superior a $20 \mathrm{~mm}$

Números de dias com a precipitação acima de nn $\mathrm{mm}$, definido pelo usuário

\section{Absoluto}

Quantidade máxima de precipitação em um dia

Quantidade máxima de precipitação em cinco dia

\section{Duração}

Dias consecutivos secos

Dias consecutivos úmidos

\author{
Rx1day Máximo anual de \\ precipitação em 1 dia \\ Máximo anual de \\ Rx5day precipitação em 5 dias \\ consecutivos
}

R10

R20

$\mathrm{mm}$
$\mathrm{mm}$

Dias

Dias

Dias $\mathrm{nn} \mathrm{mm}$, definido pelo usuário

Contagem anual de dias

Número de dias no ano com precipitação $\geq 20 \mathrm{~mm}$ $\mathrm{mm}$ $\mathrm{mm}$ Número máximo de dias CDD consecutivos com RR $\leq$ Dias $1 \mathrm{~mm}$ Número máximo de dias consecutivos com RR $\geq$ $1 \mathrm{~mm}$ 


\section{Outros}

Precipitação total anual nos dias úmidos

Índice simples de intensidade diária
Precipitação total anual

PRCPTOT nos dias úmidos ( $R R \geq$ $1 \mathrm{~mm}$ )

Precipitação total anual

SDII dividida pelo número de

dias úmidos $\mathrm{mm}$

$\mathrm{mm} \cdot \mathrm{dia}^{-1}$

Os valores padronizados para estimar os índices de extremos climáticos no programa RClimdex SU (Dias de verão), TR (Noites tropicais) e Rnn (Números de dias com a precipitação acima de um valor definido pelo usuário) foram definidos para ser aplicado em todo o globo, entretanto, o programa oferece a opção de definir os limiares pois nem sempre os valores padronizados correspondem as regiões de estudos onde são aplicados.

Por isso, neste estudo foram definidos limiares (Tabela 2) para os índices de acordo com a média aritmética mais o desvio padrão dos dados diários de cada estação, utilizando uma série temporal de 30 anos.

Tabela 2 - Limiares utilizados para os índices definidos pelo usuário.

\begin{tabular}{llccc}
\hline Bioma & Estações & SU & TR & Rnn \\
\hline \multirow{2}{*}{ Amazônia } & Bacabal & 37 & 25 & 31 \\
& São Luís & 33 & 25 & 34 \\
& Turiaçu & 33 & 25 & 31 \\
& Zé Doca & 35 & 24 & 28 \\
Cerrado & Alto Parnaíba & 36 & 23 & 26 \\
& Balsas & 36 & 24 & 28 \\
& Barra do Corda & 35 & 24 & 25 \\
& Carolina & 36 & 24 & 30 \\
& Caxias & 37 & 25 & 30 \\
& Chapadinha & 35 & 24 & 31 \\
& Colinas & 36 & 23 & 28 \\
& Imperatriz & 36 & 24 & 29 \\
\hline
\end{tabular}

Foi realizada a correlação de Spearman entre os índices de extremos climáticos de precipitação que apresentaram significância estatística $(p<0,05)$ e anomalias oceânicas do MEI, PDO e AMO.

\section{RESULTADOS}

O Painel Intergovernamental sobre Mudanças Climáticas define um extremo climático como "a ocorrência de um valor da variável climática perto das extremidades superiores (ou inferiores) dentro do intervalo de valores observados da variável" (IPCC, 2012). 
As tabelas 3 e 4 mostram as tendências dos índices extremos de temperatura e precipitação para o Estado do Maranhão, respectivamente. Os valores destacados em negrito apresentam significância estatística ao nível de $5 \%(p<0,05)$, enquanto os valores sombreados apresentam significância estatística de $10 \%(0,05<p<0,1)$. As figuras 2 e 3 mostram as tendências de extremos climáticos para temperatura e a figura 4 para precipitação no Estado do Maranhão (material complementar). 
Tabela 3 - Tendência dos índices de extremos climáticos de temperatura para o Estado do Maranhão de 1986 a 2016

\begin{tabular}{|c|c|c|c|c|c|c|c|c|c|c|c|c|}
\hline Bioma & Estações & $\begin{array}{c}\text { SU } \\
\text { (dias) }\end{array}$ & $\begin{array}{c}\text { TR } \\
\text { (dias) }\end{array}$ & $\begin{array}{l}\text { TXX } \\
\left({ }^{\circ} \mathrm{C}\right)\end{array}$ & $\begin{array}{l}\text { TXn } \\
\left({ }^{\circ} \mathrm{C}\right)\end{array}$ & $\begin{array}{l}\text { TNx } \\
\left({ }^{\circ} \mathrm{C}\right)\end{array}$ & $\begin{array}{l}\text { TNn } \\
\left({ }^{\circ} \mathrm{C}\right)\end{array}$ & $\begin{array}{c}\text { Tx90p } \\
(\%)\end{array}$ & $\begin{array}{c}\text { Tx10p } \\
(\%)\end{array}$ & $\begin{array}{c}\text { Tn90p } \\
(\%)\end{array}$ & $\begin{array}{c}\text { Tn10p } \\
(\%)\end{array}$ & $\begin{array}{l}\text { DRT } \\
\left({ }^{\circ} \mathrm{C}\right) \\
\end{array}$ \\
\hline \multirow[t]{4}{*}{ Amazônia } & Bacabal & 2.747 & 4.716 & 0.093 & 0.074 & 0.093 & 0.144 & & & & & -0.094 \\
\hline & São Luís & 3.442 & 3.479 & 0.067 & 0.021 & 0.026 & 0.042 & 1.213 & -0.605 & 0.856 & -0.063 & 0.031 \\
\hline & Turiaçu & 4.536 & 0.52 & 0.084 & 0.053 & 0 & 0.059 & 0.995 & -0.742 & 0.15 & 0.226 & 0.068 \\
\hline & Zé Doca & 0.63 & 5.785 & 0.005 & 0.045 & 0.047 & 0.081 & 0.283 & -0.066 & 1.186 & -0.82 & -0.058 \\
\hline \multirow[t]{8}{*}{ Cerrado } & Alto Parnaíba & 1.859 & 1.235 & 0.078 & 0.032 & 0.035 & 0.001 & 0.828 & -0.389 & 0.399 & -0.244 & 0.031 \\
\hline & Balsas & 2.355 & 1.67 & 0.098 & 0.079 & 0.01 & 0.065 & 1.188 & -0.537 & 0.541 & -0.245 & 0.049 \\
\hline & Barra do Corda & 3.134 & 5.743 & 0.068 & 0.022 & 0.114 & 0.119 & 1.16 & -0.513 & 1.479 & -1.007 & -0.06 \\
\hline & Carolina & 2.504 & 2.426 & 0.061 & 0.031 & 0.049 & 0.045 & 0.676 & -0.329 & 0.518 & -0.474 & 0.016 \\
\hline & Caxias & 3.401 & 1.596 & 0.147 & 0.109 & 0.057 & 0.059 & 0.838 & -1.073 & 0.634 & -0.764 & 0.038 \\
\hline & Chapadinha & 2.648 & 2.596 & 0.072 & 0.061 & 0.028 & 0.05 & 1.076 & -0.548 & 0.844 & -0.666 & 0.018 \\
\hline & Colinas & 2.196 & 2.042 & 0.085 & 0.017 & 0.02 & -0.021 & 0.955 & -0.471 & 0.419 & -0.159 & 0.04 \\
\hline & Imperatriz & 2.968 & 0.118 & 0.102 & 0.035 & 0.011 & 0.025 & 0.735 & -0.653 & 0.068 & -0.337 & 0.053 \\
\hline
\end{tabular}

Os valores destacados em negritos apresentam alta significância $(p<0,05)$ e os sombreados apresentam boa significância $(0,05<\mathrm{p}<0,1)$ 
A seguir serão apresentados os resultados relacionados aos índices de temperatura e precipitação. Serão utilizados os anos de 1986 e 2016 para demonstrar a magnitude das mudanças desses elementos climáticos no período estudado, assim como os gráficos com a evolução anual e tendência para cada índice de temperatura e precipitação que foram estatisticamente significativos (material suplementar). Os anos de 1986 e 2016 apresentaram atuação do El niño moderada e forte, respectivamente, e embora sejam anos excepcionais para identificação de padrões, um estudo realizado por Silva et al (2016), aponta que há uma tendência no aumento de temperatura no Maranhão mesmo em anos de La niña, com chuvas acima da média, como em 2009 e 2010.

\section{ANÁLISE NA DETECÇÃO DE TENDÊNCIAS CLIMÁTICAS NO PADRÃO DAS TEMPERATURAS MÁXIMAS E MÍNIMAS}

Analisando as tendências estatisticamente significativas de temperatura para o Estado do Maranhão, observa-se que o índice Dias de Verão (SU) mostrou tendências positivas estatisticamente significativas em todas as estações, exceto para a estação de Zé Doca, que também foi positiva, porém não apresentou significância estatística $(p<0,05)$. Dessa forma, há um aumento no número de dias em que a temperatura do ar foi maior do que os limiares definidos para cada estação, que variam de 33 a $37^{\circ} \mathrm{C}$.

Em 1986 na estação de Turiaçu, no bioma amazônico, em nenhum dia do ano houve registro da temperatura acima do limiar de $33^{\circ} \mathrm{C}$, entretanto no ano de 2016, houve 151 dias com registro da temperatura máxima acima de $33^{\circ} \mathrm{C}$. A estação de Caxias, no bioma cerrado, em 1986 também não registrou nenhum dia com a temperatura máxima acima do limiar de $37^{\circ} \mathrm{C}$, enquanto que no ano de 2016 foram 107 dias com registros acima de $37^{\circ} \mathrm{C}$.

O índice Noites Tropicais (TR) mostrou que a maioria das estações apresentou tendências positivas de aumento na temperatura mínima, maior que os limiares definidos para cada estação, que variam de 23 a $25^{\circ} \mathrm{C}$. Apenas as estações de Turiaçu e Imperatriz, no bioma amazônico e cerrado, respectivamente, não apresentaram tendências significativas $(p<0,05)$.

A estação de Zé Doca, bioma amazônico, em 1986 registrou apenas 1 dia em que a temperatura mínima excedeu o limiar de $24^{\circ} \mathrm{C}$. O ano de 2016 , no entanto possui 179 dias com o registro acima do limiar de $24^{\circ} \mathrm{C}$. No bioma cerrado, no ano de 1986 na estação de Barra do Corda também houve apenas 1 registro da temperatura mínima acima do limiar de $24^{\circ} \mathrm{C}$, contudo em 2016 foram 225 dias.

Em relação ao índice que representa o valor máximo mensal da temperatura máxima diária (TXX), todas as estações apresentaram tendências positivas e foram estatisticamente significativas, com exceção da estação de Zé Doca.

A estação de Bacabal, no bioma amazônico, em 1986 apresentou o valor máximo mensal da temperatura máxima diária de $38,8^{\circ} \mathrm{C}$, no mês de outubro. Em 2016, esse valor foi para $41^{\circ} \mathrm{C}$, também no mês de outubro, configurando uma tendência de aumento de $2,2^{\circ} \mathrm{C}$. No bioma cerrado, a estação de Caxias em 1986 apresentou o TXx de $36,8^{\circ} \mathrm{C}$ correspondente ao mês de outubro. No ano de 2016 não foi possível gerar o índice devido à quantidade de falhas nos 
registros, no entanto, em 2015, a temperatura máxima alcançou $41,7{ }^{\circ} \mathrm{C}$ em outubro e $42,7^{\circ} \mathrm{C}$ no mês de dezembro.

O índice do valor mínimo mensal da temperatura máxima diária (TXn) mostrou que todas as estações apresentaram tendências positivas, mas somente em 6 estações essas tendências foram estatisticamente significativas $(p<0,05)$.

Na estação de Bacabal, em 1986 o TXn foi $24,2^{\circ} \mathrm{C}$ em fevereiro. Em 2016 esse valor aumentou para $28^{\circ} \mathrm{C}$ também no mês de fevereiro, correspondendo a um aumento de $3,8^{\circ} \mathrm{C}$. Na estação de Caxias, em 1986 o TXn foi de $25,3^{\circ} \mathrm{C}$ no mês de fevereiro e em 2015 esse registro teve aumento de $1,9^{\circ} \mathrm{C}$ atingindo 27,2 ${ }^{\circ} \mathrm{C}$.

Todas estações apresentaram tendências positivas para o índice do valor máximo mensal da temperatura mínima diária $(\mathrm{TNx})$, porém em apenas 8 estações essas tendências foram estatisticamente significativas $(p<0,05)$.

Em 1988 a estação de Barra do Corda registrou o TNx de $24,2^{\circ} \mathrm{C}$, enquanto no ano de 2016 obteve o registro de $26^{\circ} \mathrm{C}$ no mês de fevereiro e $27,1^{\circ} \mathrm{C}$ no mês de novembro. Em Bacabal no ano de 1991 o TNx foi $25,4^{\circ} \mathrm{C}$ em fevereiro, em 2012 alcançou $26,4^{\circ} \mathrm{C}$ em novembro.

O mesmo comportamento foi observado para o valor mínimo mensal da temperatura mínima diária representado pelo índice TNn, indicando 8 estações com tendências positivas estatisticamente significativas 3 no bioma amazônico e 5 no cerrado.

Em 1988 na estação de Barra do Corda, agosto foi o mês que correspondeu ao TNn, com a temperatura de $14,2^{\circ} \mathrm{C}$. No ano de 2016 o mês de julho registrou o TNn de $16,5^{\circ} \mathrm{C}$. Na Estação de Bacabal no ano de 1991 o mês de julho teve o TNn, com a temperatura de $19,1^{\circ} \mathrm{C}$. No ano de 2012 o mês de janeiro registrou TNn com temperatura de $20,1^{\circ} \mathrm{C}$.

O índice que corresponde a dias quentes (Tx90p) apresentou tendências positivas estatisticamente significativas em todas as estações, com exceção da estação de Zé Doca.

Em 1987 na estação de São Luís, bioma amazônico, 1,42\% foi a porcentagem anual dos dias acima do percentil 90 para temperatura máxima, enquanto no ano de 2016 essa porcentagem aumentou para 49,36\%. No bioma cerrado, em 1986 na estação de Balsas a Tx90p foi de 0,55\%, já em 2016 essa porcentagem atingiu $57,67 \%$ dos dias.

O índice de dias frios (Tx10p) apresentou tendências negativas estatisticamente significativas em todas as estações, com exceção da estação Zé Doca.

Em 1986 na estação de São Luís a porcentagem anual dos dias abaixo do percentil 10 para temperatura máxima foi de 32,6\%, já em 2016 essa porcentagem caiu para $1,11 \%$. Enquanto na estação de Carolina, bioma cerrado, o ano de 1987 a Tx10p foi de 7,6\%, já no ano de 2016 essa porcentagem caiu para $4,6 \%$ dos dias.

Em relação ao índice das noites quentes (Tn90p), esse também apresentou tendências positivas estatisticamente significativas em todas as estações, menos na estação de Turiaçu e Imperatriz. 
Em 1987 na estação de Zé Doca, a porcentagem anual das noites acima do percentil 90 para temperatura mínima foi de 1,1\%, enquanto no ano de 2016 essa porcentagem aumentou para 35,04\%. Na estação de Barra do Corda, no ano de 1988 a Tn90p foi de 2,1\%, já no ano de 2016 essa porcentagem aumentou para $67,04 \%$ dos dias.

Todos os índices mostraram tendências negativas para as noites frias (Tn10p), exceto para a estação de Turiaçu. Contudo, apenas 6 das 12 estações apresentaram tendências estatisticamente significativas.

Em 1987 na estação de Zé Doca a porcentagem anual das noites abaixo do percentil 10 para temperatura mínima foi de $21,53 \%$, enquanto que no ano de 2016 a Tn10p caiu para 0,84\%. Na estação de Alto Parnaíba, no ano de 1988 a Tn10p foi de 19,18\%, já no ano de 2016 essa porcentagem diminuiu para $6,2 \%$ dos dias.

A estação de Bacabal foi a única estação para a qual não foi possível calcular todos os índices porque o número de falhas nos dados foi maior que $20 \%$ da série. Os índices que não foram gerados são: Tx10p, Tx90p, Tn10p, Tn90p.

A amplitude térmica diária (DTR) apresentou tendência positiva na maioria das estações, porém apenas 9 foram estatisticamente significativos ( $p<$ $0,05)$, sendo 2 negativos e 7 positivos.

Em 1986 na estação de Imperatriz, bioma cerrado, a DTR ficou em torno de $10,7^{\circ} \mathrm{C}$, já em 2013 essa diferença aumentou para 11, $4{ }^{\circ} \mathrm{C}$. Enquanto que na estação de Turiaçu, bioma amazônico, em 1986 a DTR anual foi de $6,9{ }^{\circ} \mathrm{C}$, já no ano de 2016 aumentou para $8,1^{\circ} \mathrm{C}$

Portanto, os resultados evidenciam um aumento no número de dias e noites quentes e diminuição de dias e noites frias no Maranhão, com destaque para as estações de Caxias e Chapadinha onde todos os índices apresentaram tendências significativas.

A estação de Imperatriz, apresentou um aumento em relação ao número de dias quentes, aumento no valor máximo da temperatura máxima e diminuição de dias frios. A estação de Zé Doca apresentou um aumento do número de noites quentes, aumento no valor mínimo da temperatura máxima e diminuição de noites frias. 
Tabela 4 - Tendência dos índices de extremos climáticos de Precipitação para o Estado do Maranhão de 1986 a 2016

\begin{tabular}{|c|c|c|c|c|c|c|c|c|c|c|c|}
\hline Bioma & Estações & $\begin{array}{c}\text { Rx1day } \\
(\mathrm{mm})\end{array}$ & $\begin{array}{c}\text { Rx5day } \\
\text { (mm) }\end{array}$ & $\begin{array}{l}\text { R10mm } \\
\text { (dias) }\end{array}$ & $\begin{array}{l}\text { R20mm } \\
\text { (dias) }\end{array}$ & $\begin{array}{c}\text { Rnnmm } \\
\text { (dias) }\end{array}$ & $\begin{array}{c}\text { CDD } \\
\text { (dias) }\end{array}$ & $\begin{array}{c}\text { CWD } \\
\text { (dias) }\end{array}$ & $\begin{array}{l}\text { R95p } \\
\text { (mm) }\end{array}$ & $\begin{array}{l}\text { R99p } \\
\text { (mm) }\end{array}$ & $\begin{array}{c}\text { PRCPTOT } \\
(\mathbf{m m})\end{array}$ \\
\hline nia & $\begin{array}{l}\text { Bacabal } \\
\text { São Luís } \\
\text { Turiaçu } \\
\text { Zé Doca }\end{array}$ & $\begin{array}{c}-0.221 \\
0.277 \\
0.682 \\
-0.262\end{array}$ & $\begin{array}{c}0.576 \\
-0.898 \\
0.878 \\
\mathbf{- 1 . 9 7 9}\end{array}$ & $\begin{array}{c}-0.089 \\
-0.346 \\
-0.32 \\
-0.289\end{array}$ & $\begin{array}{l}-0.242 \\
-0.262 \\
-0.225\end{array}$ & $\begin{array}{l}-0.093 \\
-0.193 \\
-0.083 \\
\mathbf{- 0 . 1 9 9}\end{array}$ & $\begin{array}{c}0.15 \\
0.945 \\
0.181 \\
\mathbf{0 . 9 9}\end{array}$ & $\begin{array}{c}0.093 \\
-0.077 \\
0.124 \\
0.037\end{array}$ & $\begin{array}{c}2.088 \\
-6.44 \\
1.109 \\
-2.073\end{array}$ & $\begin{array}{c}-0.202 \\
-0.362 \\
4.877 \\
-1.577\end{array}$ & $\begin{array}{c}-1.539 \\
-15.335 \\
-8.231 \\
-7.46\end{array}$ \\
\hline Cer & $\begin{array}{l}\text { Alto Parnaíba } \\
\text { Balsas } \\
\text { Barra do Corda } \\
\text { Carolina } \\
\text { Caxias } \\
\text { Chapadinha } \\
\text { Colinas } \\
\text { Imperatriz }\end{array}$ & $\begin{array}{c}0.137 \\
-0.366 \\
0.057 \\
0.542 \\
-0.393 \\
-0.088 \\
-0.688 \\
0.068 \\
\end{array}$ & $\begin{array}{c}0.528 \\
0.236 \\
0.15 \\
\mathbf{1 . 5 8 6} \\
-1.12 \\
0.447 \\
-1.03 \\
-0.778 \\
\end{array}$ & $\begin{array}{c}0.027 \\
-0.139 \\
-0.113 \\
0.043 \\
-0.231 \\
-0.192 \\
-0.245 \\
-0.102 \\
\end{array}$ & $\begin{array}{l}-0.098 \\
0.008 \\
-0.069 \\
0.023 \\
-0.04 \\
-0.145 \\
-0.145 \\
-0.079 \\
\end{array}$ & $\begin{array}{l}-0.072 \\
-0.01 \\
-0.015 \\
0.053 \\
-0.066 \\
-0.033 \\
-0.126 \\
\mathbf{- 0 . 2 3 5} \\
\end{array}$ & $\begin{array}{c}0.673 \\
0.302 \\
-0.141 \\
\mathbf{- 1 . 2 1 1} \\
0.791 \\
0.327 \\
-0.276 \\
0.074 \\
\end{array}$ & $\begin{array}{l}-0.025 \\
-0.067 \\
-0.013 \\
0.076 \\
-0.115 \\
-0.084 \\
-0.077 \\
-0.017 \\
\end{array}$ & $\begin{array}{c}-2.393 \\
0.243 \\
-0.237 \\
4.394 \\
-4.761 \\
3.591 \\
-\mathbf{- 7 . 3 5 5} \\
\mathbf{- 1 0 . 9 3 9} \\
\end{array}$ & $\begin{array}{c}0.333 \\
-0.192 \\
0.368 \\
3.185 \\
-3.757 \\
0.816 \\
-0.654 \\
-2.301 \\
\end{array}$ & $\begin{array}{l}-3.303 \\
-3.587 \\
-2.803 \\
4.504 \\
-7.869 \\
-4.317 \\
-10.425 \\
-10.479 \\
\end{array}$ \\
\hline
\end{tabular}

Os valores destacados em negritos apresentam alta significância $(p<0,05)$ e os sombreados apresentam boa significância $(0,05<\mathrm{p}<0,1)$ 


\section{ANÁlise NA deTECÇÃo de tendÊnCIAs CLIMÁtICAs No PADRÃo PLUVIOMÉTRICO}

De maneira geral, a precipitação se manteve constante. Apenas 4 índices indicaram tendências estatisticamente significativas ( $p<0,05)$ em algumas estações.

O índice número de dias com precipitação acima de $\mathrm{nn} \mathrm{mm}$ (Rnn mm) apresentou tendência negativa em 11 estações, mas apenas nas estações de Zé Doca e Imperatriz essas tendências foram estatisticamente significativas. A estação de Carolina foi a única que apresentou tendência positiva, mas não foi estatisticamente significativa.

Na estação de Zé Doca o ano de 1987 apresentou 11 dias com a precipitação diária acima de $28 \mathrm{~mm}$, entretanto em 2012 foram apenas 8 dias e durante 2016, 12 dias. Embora o ano de 2016 tenha apresentado mais dias acima da média de precipitação diária anual, é possível notar ao longo da série temporal uma tendência de diminuição do número de dias com a precipitação acima de $28 \mathrm{~mm}$ durante o ano na série temporal da estação de Zé Doca. Na estação de Imperatriz o ano de 1986 teve 19 dias com R29 mm, no ano de 2015 foram apenas 6 dias.

O índice quantidade máxima de precipitação em 5 dias consecutivos no mês (Rx5day) mostrou tendências de aumento em 7 estações, mas apenas a estação de Carolina foi estatisticamente significativa $(p<0,05)$ e, dentre as 5 estações que apresentaram tendência de decréscimo, apenas a estação de Zé Doca foi estatisticamente significativa $(p<0,05)$.

Na estação de Carolina, bioma cerrado, o mês de janeiro de 1986 foi o que apresentou o maior valor da Rx5day com 148,8 mm, enquanto no ano de 2016, janeiro também foi o mês que correspondeu ao maior registro do índice com 211,1 mm. Na estação de Zé Doca, bioma amazônico, em 1988 o mês de abril teve o maior registro da Rx5day com 208,7 mm, decaindo para $102 \mathrm{~mm}$ em abril de 2016. Entretanto, o mês que corresponde ao máximo anual de precipitação em 5 dias em 2016 é fevereiro com 124,7 mm.

O índice de dias muito úmidos (R95p) mostrou tendência negativa para a maioria das estações, mas apenas as estações de Imperatriz e Colinas apresentaram tendências negativas com significância estatística $(p<0,05)$. Não houve tendências estatisticamente significativas $(p<0,05)$ nas estações do bioma amazônico.

Na estação de Imperatriz, no ano de 1986 a precipitação total anual em que a precipitação diária (RR) é maior do que o percentil 95 foi de $554,5 \mathrm{~mm}$, já no ano de 2015 esse valor diminui para 70,7 mm. Na estação de Colinas no ano de 1986 a precipitação total anual RR > 95 percentil foi de $462 \mathrm{~mm}$, enquanto no ano de 2016 esse valor diminuiu para 124.

O índice de dias consecutivos secos (CDD) apresentou 9 estações com tendências positivas e $3 \mathrm{com}$ tendências negativas. Entretanto apenas nas estações de Zé Doca e Carolina essas tendências foram estatisticamente significativas ( $p<0,05)$, com tendência positiva e negativa, respectivamente.

Na estação de Carolina, o ano de 1986 teve 81 dias consecutivos secos, enquanto em 2016 esse número caiu para 44 dias. A estação de Zé Doca no ano de 1987 teve 54 dias, contudo o ano com maior número de dias consecutivos 
secos foi 2009 com 96 dias, enquanto que o ano de 2016 apresentou também 54 dias. Apesar disso, ao longo da série temporal é possível notar uma tendência de aumento no número de dias consecutivos secos na estação de Zé Doca.

\section{CORRELAÇÃo ENTRE OS ÍNDICES DE EXTREMOS CLIMÁtICOS DE PRECIPITAÇÃO E ANOMALIAS OCEÂNICAS}

A tabela 5 mostra a correlação entre os índices de extremos climáticos Rnn, R95p, Rx5day e CDD e as médias anuais das anomalias oceânicas do MEI, PDO e AMO.

Apenas as estações de Balsas, Barra do Corda e Carolina no bioma Cerrado apresentaram uma correlação estatística significativa $(p<0,05)$.

$O$ índice Rx5day apresentou correlação negativa com o MEI nas estações de Balsas e Barra do Corda e uma correlação negativa com o PDO na estação de Barra do Corda. Em relação ao AMO o índice Rx5day apresentou correlação positiva.

O índice CDD apresentou uma correlação negativa com o AMO na estação de Carolina.

Embora essas estações tenham apresentado correlações significativas o $\mathrm{R}^{2}$ foi baixo, indicando pouca influência em relação a esses eventos extremos nessas localidades. 
Tabela 5 - Correlação entre índices de extremos climáticos de precipitação e anomalias de TSM

\begin{tabular}{|c|c|c|c|c|c|c|c|c|c|c|c|c|c|}
\hline \multirow[t]{2}{*}{ Biomas } & \multirow[t]{2}{*}{ Estações } & \multicolumn{4}{|c|}{ MEI } & \multicolumn{4}{|c|}{ PDO } & \multicolumn{4}{|c|}{ AMO } \\
\hline & & Rnn & R95p & Rx5day & CDD & Rnn & R95p & Rx5day & CDD & $\mathrm{Rnn}$ & R95p & Rx5day & CDD \\
\hline \multirow[t]{4}{*}{ Amazônia } & Bacabal & -0.134 & 0.075 & -0.181 & 0.103 & -0.137 & 0.093 & -0.127 & 0.173 & 0.127 & 0.106 & 0.149 & 0.067 \\
\hline & São Luís & -0.168 & -0.057 & 0.211 & -0.106 & 0.026 & -0.033 & 0.117 & -0.135 & 0.101 & -0.062 & -0.139 & 0.223 \\
\hline & Turiaçu & 0.052 & -0.041 & -0.035 & -0.044 & 0.014 & -0.027 & 0.000 & -0.122 & 0.110 & 0.186 & 0.195 & 0.179 \\
\hline & Zé Doca & -0.034 & 0.051 & 0.020 & -0.126 & -0.032 & -0.060 & -0.020 & -0.096 & -0.124 & -0.076 & -0.188 & 0.318 \\
\hline \multirow[t]{8}{*}{ Cerrado } & Alto Parnaíba & -0.013 & 0.103 & -0.005 & 0.130 & 0.011 & 0.184 & 0.025 & 0.089 & 0.073 & 0.106 & 0.154 & 0.079 \\
\hline & Balsas & 0.020 & -0.329 & -0.425 & 0.035 & 0.049 & -0.224 & -0.194 & 0.149 & 0.023 & 0.070 & 0.170 & -0.017 \\
\hline & Barra do Corda & -0.280 & -0.306 & -0.598 & 0.086 & -0.071 & -0.195 & -0.463 & 0.246 & 0.094 & 0.045 & 0.087 & -0.002 \\
\hline & Carolina & 0.006 & 0.093 & 0.005 & 0.055 & 0.072 & 0.000 & -0.184 & 0.201 & 0.238 & 0.251 & 0.382 & -0.595 \\
\hline & Caxias & 0.115 & -0.120 & 0.038 & -0.180 & 0.201 & 0.039 & 0.082 & -0.181 & 0.119 & -0.053 & -0.153 & 0.078 \\
\hline & Chapadinha & -0.020 & 0.346 & 0.133 & -0.176 & -0.129 & 0.157 & -0.077 & -0.041 & 0.136 & 0.225 & 0.162 & 0.096 \\
\hline & Colinas & 0.087 & 0.248 & 0.105 & 0.123 & 0.071 & 0.297 & 0.098 & 0.107 & 0.056 & -0.077 & -0.259 & -0.248 \\
\hline & Imperatriz & -0.104 & 0.093 & -0.066 & 0.011 & 0.111 & 0.231 & 0.122 & 0.190 & -0.220 & -0.348 & -0.058 & 0.190 \\
\hline
\end{tabular}

Os valores destacados em negritos apresentam alta significância $(p<0,05)$ 


\section{DISCUSSÃO}

Os resultados obtidos neste artigo estão em acordo com outros estudos em escalas global, regional e local (ALEXANDER et al., 2006, POWELL e KEIM, 2015, ARAÚJO, SANTOS e NASCIMENTO, 2015).

Os índices de extremos climáticos relacionados à temperatura indicam uma forte tendência de aumento da temperatura diurna e noturna, com o aumento de dias e noites com temperaturas acima da média registrada pela normal climatológica (1986-2016).

Outros estudos também detectaram alterações no clima pelo aumento da temperatura. Barry et al. (2018), ao avaliar índices extremos em treze países na África Ocidental por 2 períodos 1960-2010 e 1981-2010, identificaram aumento significativos de $0,16{ }^{\circ} \mathrm{C} /$ década e 0,28 ${ }^{\circ} \mathrm{C} /$ década na média anual das temperaturas máxima e mínima, respectivamente para todas as estações terrestres disponíveis durante os últimos 50 anos.

Brown et al. (2010), ao realizar um estudo de mudanças nos índices de extremos climáticos no nordeste dos Estados Unidos no período de 1870 a 2015, identificaram um forte aquecimento com aumento na frequência de eventos quentes (dias quentes e noites quentes) e diminuição na frequência de eventos frios (dias frios e noites frias). Vincent et al. (2005) encontraram resultado similar ao analisar tendências em índices de extremos climáticos da temperatura diária na América do Sul (1960-2000), onde constataram um aumento significativo na temperatura mínima diária com o aumento da porcentagem das noites quentes e diminuição das noites frias.

Estudos realizados no Brasil seguem a mesma tendência com o aumento de dias e noites quentes e diminuição de dias e noites frias (SANTOS e OLIVEIRA, 2017, NATIVIDADE et al., 2017).

Os índices de precipitação apresentaram poucas tendências estatisticamente significativas e de forma variada. Na região sul do Maranhão, a estação de Carolina apresentou índices que evidenciam um aumento da umidade, já no oeste maranhense, na microrregião de Pindaré, a estação de Zé Doca indica um aumento das condições secas, mostrando um aumento do período de estiagem.

Este resultado está de acordo com o estudo realizado na região de transição Amazônia-Cerrado no Estado do Maranhão por Silva et al. (2016) que, ao analisar tendências utilizando o teste de Mann-Kendall, verificaram um aumento da precipitação na estação de Carolina e uma diminuição da precipitação no período seco na estação de Zé Doca. Almeida et al. (2017) analisou a variabilidade espaço-temporal de tendências para Amazônia Legal Brasileira e observaram que em relação a precipitação, não houve tendências significativas para a maioria das regiões, entretanto, algumas estações apresentaram tendências de aumento para as chuvas anuais, no período chuvoso e outras tendências de diminuição no período seco.

Resultados similares foram encontrados por Supari et al. (2017) na Indonésia ao analisar índices de extremos climáticos. Observaram que a precipitação se mantém constante sem muitas tendências estatisticamente significativas, porém observaram também tendências de condições mais úmidas correspondentes ao índice SDII em todo o país, e RX1day, R99p ao norte. 
Analisando sazonalmente, identificaram tendências negativas nos meses de junho, julho e agosto (JJA), setembro, outubro e novembro (SON) e março, abril e maio (MAM) na região sul.

Haylock et al. (2006), em seu estudo de tendências de precipitação total e extrema da América do Sul no período de 1960-2000 e ligações com a temperatura da superfície do mar (TSM), verificaram que o padrão de tendência da precipitação total e extrema mostraram mudança em algumas regiões para condições mais úmidas no Equador e norte do Peru e na região sul do Brasil, Paraguai, Uruguai e no norte e centro da Argentina. Também foi observado uma diminuição no sul do Peru e sul do Chile.

Da Silva et al. (2017) analisaram tendências de mudanças climáticas na precipitação pluviométrica nas bacias hidrográficas do estado de Pernambuco no período de 1962 a 2011 e observaram evidências de mudanças no clima de algumas bacias hidrográficas principais, diagnosticando indícios de aceleração no processo de aridez das bacias dos rios Ipanema, Brígida e Garças, e tendência de aumento dos eventos extremos máximos de precipitação para as bacias dos rios Mundaú, Sirinhaém e Garças.

Em relação a correlação dos índices de extremos climáticos de precipitação com as anomalias oceânicas, Aragão et al. (2018) constaram que grandes eventos de seca na Amazônia nos anos de 2005, 2010 e 2015 ocorreram quando os índices de anomalias oceânicas MEI, PDO e AMO foram positivos concomitantemente. $O$ nordeste brasileiro tem como principal manifestação da variabilidade climática, a seca, onde também está associada às variabilidades de temperatura da superfície do mar nos Oceanos Pacífico e Atlântico Tropical (MARENGO e SILVA DIAS, 2007; NOBRE et al., 2006). Entretanto, em relação à eventos de extremos climáticos, o estado do Maranhão demonstrou não receber uma influência direta desses eventos em escala global.

Os resultados evidenciam o aumento da temperatura e manutenção dos regimes pluviométricos. Dessa forma, a umidade deve apresentar tendência de altos valores, o que pode favorecer o aumento na reprodução de vetores de doenças (HOPP e FOLEY, 2003), bem como de doenças relacionadas ao saneamento básico.

No caso do Maranhão, esse quadro se agrava, pois, é o penúltimo estado no ranking de índice de desenvolvimento humano com o IDHM de 0,639, ficando atrás apenas de Alagoas com IDHM de 0,631 (BRASIL, 2013). Além disso, o Estado ocupa o $4^{\circ}$ do Brasil em rede de distribuição de água sem tratamento, com apenas 47 unidades, e o 140 lugar em relação a rede coletora de esgoto, com apenas 14 unidades (IBGE, 2008).

O Maranhão também é um dos estados que apresentam alta incidência de focos de queimadas do Brasil (http://www.inpe.br/queimadas/portal). Diante disso, com o aumento do número de dias e noites quentes, a possibilidade de queimadas pode aumentar. Estudos relatam o aumento de incêndios florestais induzidos pela seca tanto na Amazônia (ARAGÃO et al., 2018) quanto no Cerrado (MATAVELI et al., 2018), além disso, as ações antrópicas também contribuem para o aumento das queimadas, como por exemplo o desmatamento e técnicas de manejo de culturas, bem como a manutenção de pasto para gado (FEARNSIDE, 2005; COCHRANE, 2009). Associado ao aumento da temperatura, umidade e queimadas, doenças agravadas pelo clima como as respiratórias e 
cardiovasculares possuem tendência de aumento (SMITH et al, 2013; PATZ et al., 2005).

Dessa forma, diante dos estudos relatados se espera que aumente o número de incêndios, queimadas e emissões de carbono no bioma amazônico e cerrado, assim como doenças relacionadas com os extremos climáticos no Estado do Maranhão.

\section{CONCLUSÕES}

Em relação às questões climáticas focadas neste estudo, (1) estão ocorrendo extremos climáticos que configurem a mudança do clima na região de transição Amazônia-Cerrado, no Maranhão? Os resultados evidenciam que estão ocorrendo extremos climáticos na região estudada, com dias e noites mais quentes e, em algumas localidades, aumento da umidade, aumento e diminuição no volume de chuvas.

2) Com que magnitude a temperatura e precipitação estão configurando as tendências de mudança climática? Foi observado o aumento de dias com a temperatura máxima diária acima dos limiares que variam entre $33^{\circ} \mathrm{C}$ a $37^{\circ} \mathrm{C}$, bem como a ocorrência de noites mais quentes, com aumento de dias com temperaturas mínimas superiores aos limiares $23^{\circ} \mathrm{C}$ a $25^{\circ} \mathrm{C}$. Em relação à precipitação houve tendência de decréscimo nas estações de Zé Doca e Imperatriz relativo aos limiares $25 \mathrm{~mm}$ a $34 \mathrm{~mm}$ diários. Em relação à umidade, foi observado tendência de aumento em Carolina e decréscimo em Zé Doca.

3) Existe um padrão espacial definido para as mudanças climáticas nesta região estudada? Foi observado um padrão espacial, pois houve aumento das temperaturas em todas as estações estudadas. A precipitação e umidade não demonstraram um padrão bem definido, com apenas uma estação apresentando aumento no volume de chuvas e umidade e outra de decréscimo com valores estatisticamente significativos. Entretanto, os índices mostraram uma grande variação espaço-temporal em magnitude, evidenciando que o clima está mudando de forma diferente em diferentes localidades no Estado do Maranhão.

4) A ocorrência de eventos climáticos extremos é influenciada por anomalias da temperatura da superfície do mar nos oceanos Pacífico e Atlântico? Os resultados não evidenciaram um padrão de correlação entre os eventos extremos na região estudada e as anomalias da temperatura da superfície do mar nos oceanos Pacífico e Atlântico.

\section{REFERÊNCIAS}

ALEXANDER, L. V. ALEXANDER, L.V.; ZHANG, X.; PETERSON, T.C.; CAESAR, J.; GLEASON, B.; TANK, A.M.G.K.; HAYLOCK, M.; COLLINS. D; TREWIN, B.; RAHIMZADEH, F.; TAGIPOUR, A.; KUMAR, K.R.; REVADEKAR. J.; GRIFFITHS, G.; VINCENT, L; STEPHENSON, D.B.; BURN, J.; AGUILAR, E.; BRUNET, M.; TAYLOR, M.; NEW, M.; ZHAI, P.; RUSTICUCCI, M.; VAZQUEZ-AGUIRRE, J.L. Global observed changes in daily climate extremes of temperature and precipitation. Journal of Geophysical Research: Atmospheres, v. 111, n. D5, 2006. 
ALMEIDA, C.T., OLIVEIRA-JÚNIOR, J.F., DELGADO, R.C., CUBO, P., e RAMOS, M. C. Spatiotemporal rainfall and temperature trends throughout the Brazilian Legal Amazon, 1973-2013. International Journal of Climatology, v. 37, n. 4, p. 20132026, 2017.

ALVARES, C. A.; STAPE, J. L.; SENTELHAS, P. C.; GONÇALVES, J. L. M.; SPAROVEK, G. Köppen's climate classification map for Brazil. Meteorologische Zeitschrift, v. 22, n. 6, p. 711-728, 2013.

ARAGÃO, L.E.O.C.; ANDERSON, L.O.; FONSENCA, M.G.; ROSAN, T.M.; VEDOVATO, L.B.; WAGNER, F.H.; SILVA, C.V.J.; SILVA JUNIOR, C.H.L.; ARAI, E.; AGUIAR, A.P.; BARLOW, J.; BERENGUER, E.; DEETER, M.N.; DOMINGUES, L.G.; GATTI, L.; GLOOR, M.; YADVINDER, M.; MARENGO, J.A.; MILLER, J.B.; PHILLIPS, O.L.; SAATCHI, S. 21st Century drought-related fires counteract the decline of Amazon deforestation carbon emissions. Nature communications, $v$. 9, n. 1, p. 536, 2018.

ARAÚJO, F.R.C.D.; SANTOS, C.A.C; NASCIMENTO, F.C.A. Study of temperature extreme indices over hydrographic basin of low Colorado river-USA. Revista Brasileira de Meteorologia, v. 30, n. 1, p. 29-36, 2015.

ASSIS, J.; LACERDA, F.F.; DO CARMO SOBRAL, M. Análise de detecção de tendências no padrão pluviométrico na bacia hidrográfica do Rio Capibaribe. Revista Brasileira de Geografia Física, v. 5, n. 2, p. 320-331, 2012.

ASSIS, J.M.O.; SOUZA, W.M.; SOBRAL, M.C.M. Climate analysis of the rainfall in the lower-middle stretch of the São Francisco river basin based on the rain anomaly index. Revista Brasileira de Ciências Ambientais, v. 2, p. 188-202, 2015.

BARRY, A.A.; CAESAR, J.; KLEIN TANK, A.M.G.; AGUILAR, E.; MCSWEENEY, C.; CYRILLE, A.M.; NIKIEMA, M.P.; NARCISSE, K.B.; SIMA, F.; STAFFORD, G.; TOURAY, L.M.; AYILARI-NAA, J.A.; MENDES, C.L.; TOUNKARA, M.; GAR-GLAHN, E.V.S.; COULIBALY, M.S.; DIEH, M.F.; MOUHAIMOUNI, M.; OYEGADE, J.A., SAMBOU, E.; LAOGBESSI, E.T. West Africa climate extremes and climate change indices. International Journal of Climatology, 2018.

BRASIL, A. Atlas do Desenvolvimento humano no Brasil 2013. Ranking. Disponível em: http://www.atlasbrasil.org.br/2013/pt/ranking/. Acesso em: 19 fev. 18.

BROWN, P.J.; BRADLEY, R.S.; KEIMIG, F.T. Changes in extreme climate indices for the northeastern United States, 1870-2005. Journal of Climate, v. 23, n. 24, p. 6555-6572, 2010.

BÜRGER, G., MURDOCK, T.Q.; WERNER, A.T.; SOBIE, S.R.; e CANNON, A.J. Downscaling extremes-An intercomparison of multiple statistical methods for present climate. Journal of Climate, v. 25, n. 12, p. 4366-4388, 2012.

COCHRANE, M. A. Tropical Fire Ecology: Climate change, Land use and Ecosystem Dynamics. Praxis Plublishing Ltd, Chichester, UK, 2009.

EMPRESA BRASILEIRA DE PESQUISA AGROPECUÁRIA - EMBRAPA. Relatório do diagnóstico do macrozoneamento ecológico-econômico do Estado do Maranhão. BATISTELLA, M; BOLFE, E. L; VICTORIA, D. DE C.; ARAUJO, L. S. (Org.). Campinas, SP: Embrapa, 2013. 325 p. 
Instituto Brasileiro de Geografia e Estatística - IBGE. Diretoria de Pesquisas. Coordenação de População e Indicadores Sociais. Pesquisa Nacional de Saneamento Básico, 2008.

Instituto Brasileiro de Geografia e Estatística - IBGE. Biomas e sistema costeiromarinho do Brasil: compatível com a escala 1:250 000 / IBGE, Coordenação de Recursos Naturais e Estudos Ambientais. - Rio de Janeiro: IBGE, 2019. 168p. (Relatórios metodológicos, INSS 0101-2843; v. 45).

IPCC: Managing the Risks of Extreme Events and Disasters to Advance Climate Change Adaptation. A Special Report of Working Groups I and II of the Intergovernmental Panel on Climate Change. [Field, C.B., V. Barros, T.F. Stocker, D. Qin, D.J. Dokken, K.L. Ebi, M.D. Mastrandrea, K.J. Mach, G.-K. Plattner, S.K. Allen, M. Tignor, and P.M. Midgley (eds.)]. Cambridge University Press, Cambridge, UK, and New York, NY, USA, 2012, 582 pp.

IPCC: Climate Change 2013: The Physical Science Basis. Contribution of Working Group I to the Fifth Assessment Report of the Intergovernmental Panel on Climate Change. [Stocker, T.F., D. Qin, G.-K. Plattner, M. Tignor, S. K. Allen, J. Boschung, A. Nauels, Y. Xia, V. Bex and P.M. Midgley (eds.)]. Cambridge University Press, Cambridge, United Kingdom and New York, NY, USA, 2013, $1535 \mathrm{p}$.

FERREIRA, A. G, MELLO, N. G. S. Principais Sistemas Atmosféricos atuantes sobre a região Nordeste do Brasil e a influência dos Oceanos Pacífico e Atlântico no clima da região. Revista Brasileira de Climatologia. Volume 1 n. 1 dez/2005, p. 15-28.

GOCIC, M.; TRAJKOVIC, S. Analysis of changes in meteorological variables using Mann-Kendall and Sen's slope estimator statistical tests in Serbia. Global and Planetary Change, v. 100, p. 172-182, 2013.

GUMMADI, S.; RAO, K.P.C.; SEID, J.; LEGESSE, G.; KADIYALA, M. D. M.; TAKELE, R.; AMEDE, T.; WHITBREAD, A. Spatio-temporal variability and trends of precipitation and extreme rainfall events in Ethiopia in 1980-2010. Theoretical and Applied Climatology, p. 1-14, 2017.

GUO, J.; SU, T.; LI, Z.; MIAO, Y.; LI, J.; LIU, H.; XU, H.; CRIBB, M.; ZHAI, P. Declining frequency of summertime local-scale precipitation over eastern China from 1970 to 2010 and its potential link to aerosols. Geophysical Research Letters, v. 44, n. 11, p. 5700-5708, 2017.

KATZ, R.W.; BROWN, B.G. Extreme events in a changing climate: variability is more important than averages. Climatic change, v. 21, n. 3, p. 289-302, 1992.

KENDALL, M.G. Rank correlation methods, 2 ed, New York: Hafner, 1975.

KUNWAR, S.B.; BOHARA, A.K. Climate sensitivities and farmland values in Nepal: A spatial panel Ricardian approach. Journal of Development and Agricultural Economics, v. 9, n. 6, p. 145-161, 2017.

HASAN, H.; SALLEH, N.H.M. Climate change risk reduction through readiness: An assessment of extreme temperature indices for Peninsular Malaysia. Geografia-Malaysian Journal of Society and Space, v. 12, n. 10, 2017.

HAYLOCK, M.R.; PETERSON, T.C.; ALVES, L.M.; AMBRIZZI, T.; ANUNCIAÇÃO, Y.M.T.; BAEZ, J.; BARROS, V.R.; BERLATO, M.A.; BIDEGAIN, M.; CORONEL, G.; 
CORRADI, V.; GARCIA, V.J.; GRIMM, A.M.; KAROLY, D.; MARENGO, J. A.; MARINO, M.B.; MONCUNILL, D.F.; NECHET, D.; QUINTANA, J.; REBELLO, E.; RUSTICUCCI, M.; SANTOS, J.L.; TREBEJO, I.; VINCENT, L. A. Trends in total and extreme South American rainfall in 1960-2000 and links with sea surface temperature. Journal of climate, v. 19, n. 8, p. 1490-1512, 2006.

HOPP, M.J.; FOLEY, J.A. Worldwide fluctuations in dengue fever cases related to climate variability. Climate Research, v. 25, n. 1, p. 85-94, 2003.

LÜ, M., JIANG, Y., CHEN, X., CHEN, J., WU, S., \& LIU, J. Spatiotemporal Variations of Extreme Precipitation under a Changing Climate in the Three Gorges Reservoir Area (TGRA). Atmosphere, v. 9, n. 1, p. 24, 2018.

MANN, H.B., Nonparametric test against trend. Econometria, v. 13, 1945.

Maranhão (Estado). Secretaria de Estado do Meio Ambiente e Recursos Naturais. Atlas do Maranhão. Gerência de Planejamento e Desenvolvimento Econômico/ Laboratório de Geoprocessamento - UEMA. 2. ed. São Luís: GEPLAN, 2002. 44 p.

MARENGO, J. A.; DIAS, P. S. Mudanças climáticas globais e seus impactos nos recursos hídricos. Águas doces no Brasil: capital ecológico, uso e conservação, v. 3, p. 63-109, 2006.

MATAVELI, G.A.V.; SILVA, M.E.S.; PEREIRA, G.; CARDOZO, F.S.; KAWAKUBO, F.S.; BERTANI, G.; COSTA, J.C.; RAMOS, R.C.; SILVA, V.V. Satellite observations for describing fire patterns and climate-related fire drivers in the Brazilian savannas. Natural Hazards and Earth System Sciences, v. 18, n. 1, p. 125, 2018.

MUTIIBWA, D.; VAVRUS, S.J.; MCAFEE, S.A.; ALBRIGHT, T.P. Recent spatiotemporal patterns in temperature extremes across conterminous United States. Journal of Geophysical Research: Atmospheres, v. 120, n. 15, p. 73787392, 2015.

NATIVIDADE, U.A.; GARCIA, S.R.; TORRES, R.R. Trend of Observed and Projected Extreme Climate Indices in Minas Gerais State. Revista Brasileira de Meteorologia, v. 32, n. 4, p. 600-614, 2017.

NAVEAU, P.; NOGAJ, M.; AMMANN, C.; YIOU, P.; COOLEY, D.; JOMELLI, V. Statistical methods for the analysis of climate extremes. Comptes Rendus Geoscience, v. 337, n. 10-11, p. 1013-1022, 2005.

NOBRE, P.; MARENGO, J.A.; CAVALCANTI, I.F.A.; OBREGON, G. Seasonal-todecadal predictability and prediction of South American climate. Journal of climate, v. 19, n. 23, p. 5988-6004, 2006.

PATZ, J.A.; CAMPBELL-LENDRUM, D.; HOLLOWAY, T.; FOLEY, J.A. Impact of regional climate change on human health. Nature, v. 438, n. 7066, p. 310, 2005.

PEDRON, I.T.; SILVA DIAS, M.A.; DE PAULA DIAS, S.; CARVALHO, L.; FREITAS, E.D. Trends and variability in extremes of precipitation in Curitiba-Southern Brazil. International Journal of Climatology, v. 37, n. 3, p. 1250-1264, 2017.

POWELL, E.J.; KEIM, B.D. Trends in daily temperature and precipitation extremes for the Southeastern United States: 1948-2012. Journal of Climate, v. 28, n. 4, p. 1592-1612, 2015. 
RAMSAR SITES INFORMATION SERVICE. The List of Wetlands of International Importance. Disponível em: $<$ https://www.ramsar.org/sites/default/files/documents/library/sitelist.pdf>. Acesso em 18 de maio de 2018.

ROBINNE, F.N.; BLADON, K.D.; MILLER, C.; PARISIEN, M.A.; MATHIEU, J.; FLANNIGAN, M.D. A spatial evaluation of global wildfire-water risks to human and natural systems. Science of the Total Environment, v. 610, p. 1193-1206, 2018.

SANTOS, C.A.C.; OLIVEIRA, V.G.. Trends in Extreme Climate Indices for Pará State, Brazil. Revista Brasileira de Meteorologia, v. 32, n. 1, p. 13-24, 2017.

SILVA, F.B.; SANTOS, J.R.N.; FEITOSA, F.E.C.S.; SILVA, I.D.C.; ARAÚJO, M.L.S.; GUTERRES, C.E.; SANTOS, J.S.; RIBEIRO, C.V.; BEZERRA, D.S.; NERES, R.L. Evidências de Mudanças Climáticas na Região de Transição AmazôniaCerrado no Estado do Maranhão. Revista Brasileira de Meteorologia, v. 31, n. 3, p. $330-336,2016$.

SHI, J., CUI, L., WEN, K., TIAN, Z., WEI, P., ZHANG, B. Trends in the consecutive days of temperature and precipitation extremes in China during 1961-2015. Environmental research, v. 161, p. 381-391, 2018.

DA SILVA, R.O.B.; MONTENEGRO, S.M.G.L.; DE SOUZA, W.M. Tendências de mudanças climáticas na precipitação pluviométrica nas bacias hidrográficas do estado de Pernambuco. Engenharia Sanitária e Ambiental, v. 22, n. 3, 2017.

SMITH, L.T.; ARAGAO, L.E.; SABEL, C.E.; NAKAYA, T. Drought impacts on children's respiratory health in the Brazilian Amazon. Scientific reports, v. 4, p. 3726, 2014.

TANGANG, F.; JUNENG, L.; ALDRIAN, E. Observed changes in extreme temperature and precipitation over Indonesia. International Journal of Climatology, v. 37, n. 4, p. 1979-1997, 2017.

VICENT, L.A.; PETERSON, T.C.; BARROS, V.R.; MARINO, M.B.; RUSTICUCCI, M. ; CARRASCO, G.; RAMIREZ, E.; ALVES, L.M.; AMBRIZZI, T.; BERLATO, M.A.; GRIMM, A.M.; MARENGO, J.A.; MOLION, L.; MONCUNILL, D.F.; REBELLO, E.; ANUNCIAÇÃO, Y.M.T.; QUINTANA, J.; SANTOS, J.L.; BAEZ, J.; CORONEL, D.; GARCIA, J.; TREBEJO, I.; BIDEGAIN, M.; HAYLOCK, M.R.; KAROLY, D. Observed trends in indices of daily temperature extremes in South America 1960-2000. Journal of climate, v. 18, n. 23, p. 5011-5023, 2005.

WORLD METEOROLOGICAL ASSOCIATION. The global climate 2001-2010: A decade of climate extremes, summary report. Geneva, Switzerland: WMO, 16p, 2013.

YANG, C.; XU, J. A semi-parametric regression approach to climatological quantile estimation for generating percentile-based temperature extremes indices. Atmospheric Science Letters, v. 18, n. 2, p. 60-66, 2017.

Ye, J.S.; Gong, Y.H.; Zhang, F.; Ren, J.; Bai, X.K.; Zheng, Y. Which Temperature and Precipitation Extremes Best Explain the Variation of Warm versus Cold Years and Wet versus Dry Years?. Journal of Climate, v. 31, n. 1, p. 45-59, 2018. 
ZHANG, X.; YANG, F. RClimDex (1.0) user manual. Climate Research Branch Environment Canada, v. 22, 200 